\title{
COSMETIC OUTCOMES IN BREAST ONCOPLASTY: RETROSPECTIVE COHORT TO COMPARE OBJECTIVE AND SUBJECTIVE METHODS OF EVALUATION
}

Ana Paula B. Carvalho1', Régis R. Paulinelli1, Thauana D. Santos', Ruffo Freitas-Junior¹, Aloísio Garcia', Luis F. Jubé1, Rosemar M. S. Rahal', Luis Fernando P. Oliveira'

'Universidade Federal de Goiás, Goiás Association to Cancer Combat - Goiânia (GO), Brazil.

Objective: Different subjective and objective methods are available for the evaluation of the cosmetic outcome in breast reconstruction. The objective was to compare the correlation among different objective methods (BCCT and BAT), to the subjective medical impression (Harvard scale), and the subjective patient's opinion (BREAST Q). Methods: A total of 509 breast cancer patients were included in a multicentric retrospective cohort, submitted to breast reconstruction. After signing the informed consent, patients answered the BREAST Q questionnaire, information was collected from their charts and photos were taken to evaluate the results. The photos were analyzed in the BAT and BCCT.core programs, and by 3 mastologists, through the Harvard scale. Data were collected between January/2015 - February/2019. Statistical analysis and the Pearson correlation coefficient were calculated using the SPSS software. Results: The mean age of the patients was 55.8 years. Overall, 273 women (53.6\%) had been submitted to partial breast reconstruction and 236 (46.4\%) to total breast reconstruction. There was one single procedure in 321 (63.1\%) patients. The mean follow-up time was $66.3 \pm 55.0$ months. Results were good or excellent in 338 (66.4\%) cases in the surgeon's opinion, in 368 (72.3\%) patients using BCCT, and in 447 (87.8\%) using BAT (cutoff of 7). According to BREASTQ, the mean scores of satisfaction with breasts was 71.1 ( \pm 17.9 ); with results, 87.1 ( \pm 17.3$)$; physical well-being, $66.7( \pm 14.3)$; psychosocial well-being, $78.7( \pm 18.7)$, and $67.4( \pm 22.7)$ for the sexual well-being. The correlations between the BAT, the BCCT.core software programs and Harvard scale were moderate to strong, without superiority among them. There was a significant weak correlation of the two software programs, and the Harvard scale with some of the BREASTQ domains. Conclusion: The correlations among the two software and the surgeons' opinions are similarly moderate. They have an equivalent weak correlation to some of the BREASTQ domains. 